\title{
PERFIL EPIDEMIOLÓGICO DA COMUNIDADE ACADÊMICA DO IFRN EM RELAÇÃO À COVID-19 E ANÁLISE DOS FATORES DE RISCO PARA O RETORNO PRESENCIAL ÀS AULAS
}

\author{
L. M. B. M. FERREIRA ${ }^{1}$, D. S. V. D. MORAIS ${ }^{2}$, V. R. C. OLIVEIRA ${ }^{3}$, T. A. R. NASCIMENTO ${ }^{4}$, P. K. M. SILVA \\ Instituto Federal de Educação Ciência e Tecnologia do Rio Grande do Norte \\ ORCID ID: https://orcid.org/0000-0002-2191-8641 ${ }^{1}$ \\ lidiane.ferreira@ifrn.edu.br ${ }^{1}$
}

Submetido 29/09/2020 - Aceito 23/11/2020

DOI: $10.15628 /$ holos.2020.11247

\section{RESUMO}

Este trabalho é o fruto de uma pesquisa realizada no Instituto Federal de Educação, Ciência e Tecnologia do Rio Grande do Norte durante o período de suspensão das atividades presenciais em decorrência da pandemia da COVID-19, com o objetivo de traçar o perfil da comunidade acadêmica referente aos fatores de risco para as formas graves de COVID-19, assim como utilizar seus dados como estratégia para planejamento do retorno presencial às atividades. Foi realizado um levantamento por meio de questionário eletrônico e os dados analisados de forma descritiva. Um total de 14.484 pessoas responderam ao questionário, no período de 9 de junho a 21 de julho de 2020. Dentre estes, 1,71\% já haviam apresentado COVID-19 confirmada, 11,96\%
\end{abstract}

tinham apresentado sintomas sugestivos de COVID-19, $9,55 \%$ se enquadravam nos grupos considerados de risco e $61,25 \%$ residiam com alguém de grupo de risco. Como conclusão do estudo, identificamos uma baixa taxa de positividade para a doença entre os respondentes, entretanto um risco elevado de contaminação quando do retorno às atividades presenciais, visto que quase metade transita entre a cidade de residência e de trabalho/estudo, além de que a maioria mora com mais de três pessoas em sua residência. Também concluímos que os servidores estão representados como o grupo de maior risco para as formas graves de COVID-19 e que a obesidade representa uma importante condição médica, associada a vários sintomas da virose.

PALAVRAS-CHAVE: Infecções por Coronavírus, Instituições Acadêmicas, Transmissão de Doença Infecciosa.

\section{EPIDEMIOLOGICAL PROFILE OF THE IFRN ACADEMIC COMMUNITY IN RELATION TO COVID-19 AND ANALYSIS OF RISK FACTORS FOR PRESENTIAL RETURN TO FACE-TO- FACE CLASSROOMS}

\begin{abstract}
This work results from a research carried out at Instituto Federal de Educação, Ciência e Tecnologia do Rio Grande do Norte during the period of suspension of face-to-face activities due to the COVID-19 pandemic, in order to outline the profile of the academic community regarding the risk factors for severe forms of COVID-19, as well as using the data as a strategy for planning the face-to-face return to activities. A survey was carried out through an electronic questionnaire and the data analyzed descriptively. A total of 14,484 people answered the questionnaire, from June 9 to July 21, 2020. The results show that $1.71 \%$ had already confirmed COVID-19, $11.96 \%$ had presented symptoms suggestive of COVID-
\end{abstract}

$19,9.55 \%$ belonged to risk groups and $61.25 \%$ lived with someone from a risk group. As a conclusion of the study, we identified a low rate of positive cases for the disease among respondents, however a high risk of contamination when returning to face-to-face activities, since almost half transit between the city of residence and work / study, in addition to most live with more than three people in their home. We also concluded that public servers are represented as the group with the highest risk for severe forms of COVID-19 and that obesity represents an important medical condition, associated with various symptoms of the virus.

KEYWORDS: Coronavirus Infections, Schools, Disease Transmission. 


\section{INTRODUÇÃO}

Descoberta no final de 2019, na cidade de Wuhan, na China, onde foram registrados os primeiros casos, a COVID-19, causada pelo vírus Sars-CoV-2, provocou uma série de mudanças bruscas e inúmeros questionamentos a partir da dispersão da doença pelo planeta. O processo de disseminação foi rápido, em 30 de janeiro de 2020 a Organização Mundial da Saúde (OMS) declarou que o surto da doença causada pelo novo coronavírus (COVID-19) se constituía numa Emergência de Saúde Pública de Importância Internacional. Mais tarde, em 11 de março de 2020, a emergência foi alçada ao nível de pandemia e o olhar se tornou ainda mais atento para as informações acerca do assunto (Organização Panamericana de Saúde, 2020; Ministério da Saúde, 2020).

O vírus apresenta um elevado potencial de disseminação, propagando-se por meio de gotículas de saliva, secreções orais e nasais de pessoas infectadas, além de contato com objetos e superfícies contaminadas (Ministério da Saúde, 2020) e de acordo com a OMS, a maior parte dos pacientes acometidos pela doença poderão apresentar-se assintomáticos ou oligossintomáticos, e cerca de $20 \%$ dos casos necessitarão de atendimento hospitalar.

Muitos segmentos foram afetados pelo quadro de rápida disseminação da doença e os impactos puderam ser sentidos nas relações sociais, culturais, econômicas e educacionais. (Arruda, 2020). Uma dessas áreas foi a educação. Os efeitos da pandemia na educação brasileira começam a ser sentidos a partir de 6 de fevereiro de 2020, quando foi sancionada a Lei no 13.979, que dispõe sobre as medidas para enfrentamento da emergência de saúde pública de importância internacional decorrente do coronavírus responsável pelo surto de 2019/2020. Dentre as medidas a serem adotadas, encontra-se o isolamento e a quarentena para toda a população brasileira como forma de contenção na disseminação do coronavírus (Brasil , 2020). No âmbito do estado do Rio Grande do Norte, as atividades escolares presenciais foram suspensas ainda no mês de março, conforme Decreto № 29.583, de 1 o de abril de 2020, sem previsão para o seu restabelecimento (Governo do Estado do Rio Grande do Norte, 2020).

Tal preocupação com o potencial de transmissão da doença no ambiente escolar ocorre, segundo Arruda (2020), porque o ambiente escolar representa um local de elevada transmissibilidade, ocasionada pelo vínculo estabelecido entre aqueles que possuem menor grau de probabilidade e os que possuem maior grau de probabilidade de apresentar estado de gravidade da doença. O público escolar (crianças e adolescentes) tem se enquadrado como grupo com menor probabilidade de agravamento da doença, porém o ambiente acadêmico é caracterizado pela sua heterogenicidade e sua composição, formada por professores, alunos e profissionais que fazem parte deste ambiente, proporcionam uma relação de vínculo e contato diário entre si. Esta relação interpessoal entre a comunidade acadêmica e o convívio pessoal e particular desta comunidade com os seus familiares, torna os componentes deste grupo vetores em potencial para disseminação da doença COVID-19. Por esta razão, os olhares têm se tornado atentos para as escolas e a análise quanto ao retorno presencial de suas atividades e o tema tem se tornado motivo de discussão na sociedade, por entender-se que manter as adequações sanitárias recomendadas, como distanciamento, por exemplo, configura-se como uma tarefa difícil neste ambiente. 
Dentro desta seara, no âmbito do Estado do Rio Grande do Norte, o Instituto Federal de Educação, Ciência e Tecnologia do Rio Grande do Norte (IFRN) configura-se como uma instituição de educação profissional e tecnológica, sendo o ensino a sua principal finalidade. $O$ ambiente escolar do IFRN é um espaço de interação social com troca de vivências, construção de laços afetivos e saberes. Esta interação e convivência diária da comunidade acadêmica facilitam a ocorrência de aglomerações, fazendo deste ambiente um local de potencial contaminação e disseminação de micro-organismos patogênicos.

A comunidade acadêmica do IFRN é composta por discentes, servidores, estagiários e profissionais contratados que contribuem com a execução das atividades neste ambiente, os quais atuam em 22 Campi localizados em 18 municípios diferentes no RN. Trata-se da segunda maior rede de educação em capilaridade no estado, ficando atrás apenas da rede pública estadual de ensino. Diante da diversidade na composição da comunidade acadêmica do IFRN, com variações em faixa etária, sexo e condições socioeconômicas, por exemplo, faz-se necessário um olhar atento para as particularidades de cada indivíduo dentro do cenário da pandemia da COVID-19 e no ambiente da instituição.

Diante desta situação, este estudo tem o objetivo de fazer uma análise epidemiológica da situação da comunidade acadêmica do IFRN em relação ao contágio da COVID-19, assim como identificar os cenários de risco para o retorno presencial das atividades acadêmicas, os quais implicarão em tomadas de decisão a serem consideradas para o combate à pandemia no ambiente escolar. Se justifica, portanto, pelo papel enquanto instituição de ensino, em zelar pela segurança sanitária e saúde da comunidade acadêmica, bem como, de toda a sociedade, direta e indiretamente.

\section{ENDEMIA, EPIDEMIA E A PANDEMIA DA COVID-19}

Com o intuito de um melhor entendimento sobre a especificidade do tema aqui tratado, apresenta-se a diferença conceitual, bem como algumas exemplificações presentes na história sobre os conceitos em referência.

\subsection{Endemia:}

No Brasil convencionou-se designar determinadas doenças, a maioria delas parasitárias ou transmitidas por vetor, como "endemias", "grandes endemias" ou "endemias rurais". (Silva, 2003). Dessa forma, ao se falar em endemia faz-se referência a uma doença que se apresenta com duração contínua, particularmente em um espaço limitado, numa determinada região. Logo, a sua causalidade é local, podendo estar relacionada a fatores como: o clima, a forma de sociabilidade, as condições higiênicas e biológicas, e, portanto, não tendo existência de casos nem aparecendo em outras comunidades.

No Brasil há incidência de inúmeras áreas endêmicas. Doenças como a Malária, Febre Amarela, Leishmanioses, Cólera (com maior incidência nos estados da Região Norte) e Dengue (Maior incidência nos Estados da Região Nordeste), configuram-se apenas como algumas das doenças consideradas endêmicas no país (Merchán-Hamann, 1997). 
Cabe ressaltar que alguns fatores como a situação econômica, saneamento básico precário, água não tratada, poluição, alterações climáticas, culturais, sociais e biológicas podem suscitar o surgimento de uma endemia. Portanto, quando a sociedade ainda não encontra cientificamente, socialmente, economicamente e culturalmente os meios e as formas de contenção das endemias, estas podem vir a ocasionar epidemias que impactam de forma severa a saúde pública e de modo mais direto podem trazer como consequência para a vida das pessoas, muitas mortes.

\subsection{Epidemia:}

Os relatos acerca da existência de epidemias datam da antiguidade. Ao longo da história, pode-se identificar que o agravamento das epidemias coincidia com os momentos de crise da sociabilidade e com a mudança do modelo de produção (ex.: feudal, capitalista) adotado por estas mesmas sociedades. Com a advento do modo de produção capitalista, evidenciou-se o surgimento de epidemias de grandes proporções tais como: a peste Bubônica, também conhecida como epidemia da Peste Negra, ocorrida na Europa no século 14 (estima-se que já naquela época, esta doença pode ter sido responsável pela redução da população mundial de 450 milhões de pessoas para 350 milhões); Cólera, identificada em 1817, como sendo uma epidemia de grandes proporções, já naquela ocasião, vitimou centenas de milhares de pessoas; e a gripe Espanhola, ocorrida em 1918, causada pelo vírus influenza e que chegou a infectar mais de um quarto da população mundial e a dizimou de 40 a 50 milhões de pessoas (Rodrigues, 2020).

Para se descrever a caracterização de uma epidemia, torna-se necessário não se perder de vista tanto a sua expressão coletiva - uma vez que esta altera significativamente o modo de vida de grandes grupos de pessoas e em vários lugares concomitantemente, quanto a sua expressão particular - aqui cabe recorrer ao período de tempo e espaço em que se encontra ocorrendo. Em conformidade com Barata (1987), as práticas de intervenção utilizadas para o combate às epidemias refletem, de um lado, o conhecimento que se tem do fenômeno e de outro, as formas de atuação do Estado em cada período histórico.

As formas de atuação do Estado vieram se materializando ao longo do tempo na perspectiva da existência direta de um nexo entre as condições socioeconômicas e a saúde da população e apresentando variações que consistem em: profilaxia de higiene em espaços públicos e privados, medidas de isolamento social, quarentena, obrigatoriedade de incineração ou sepultamento com protocolos específicos, imunização, dentre outras. Barata (1987) elucida, ainda, que a epidemia é um fato único devido à especificidade do contexto no qual ela ocorre.

À luz deste entendimento, também se pretende analisar no próximo item, as articulações e implicações advindas das consequências de uma epidemia quando esta passa a tomar grandes proporções, do tipo em que nações inteiras são atingidas e pegas desprevenidas, o que necessariamente as leva a ter que pensar o problema em todos os seus aspectos e aqui referenciase tal como Harvey, et al. (2020) que uma epidemia se torna complexa pelo fato de ser sempre um ponto de articulação entre as determinações naturais e sociais. Sua análise completa é transversal: é preciso compreender os pontos em que as duas determinações se interceptam e tirar conclusões. 
Assim, passaremos no próximo item a descrever sobre a pandemia da COVID-19 e suas implicações a nível de grupos de riscos, transmissibilidade e a suscitação da necessidade de estratégias com vistas a prevenção e mitigação dos riscos de transmissão.

\subsection{A Pandemia da COVID-19:}

Pandemia é o termo utilizado quando uma epidemia se espalha por diferentes continentes com transmissão sustentada de pessoa a pessoa. Segundo a OMS, é a disseminação mundial de uma nova doença (World Health Organization, 2010). Como já citado na introdução, a COVID-19 é uma doença nova, que rapidamente alastrou-se pelo mundo, causando desde sintomas gripais leves até a síndrome respiratória aguda grave.

A doença é provocada por um vírus da família Coronaviridae, responsável por uma variedade de doenças no homem e nos animais, especialmente no trato respiratório. Sete espécies podem infectar humanos, sendo que três podem produzir doenças graves: o Sars-CoV-2, o Sars-CoV, agente da pandemia de Sars (síndrome respiratória aguda grave) de 2002-2003 e o Mers-CoV, causador da Mers (síndrome respiratória do Oriente Médio). Os coronavírus HKU1, NL63, OC43 e 229E estão associados a doenças com sintomatologia leve. A atual pandemia que estamos vivendo se dá pela ação do Sars-CoV-2, o mais virulento de todos. (Jornal da USP, 2020)

Os principais sintomas associados são leves, tais como febre, cansaço, tosse seca, congestão nasal, cefaleia, dor de garganta, perda do olfato e paladar, diarreia, erupção cutânea e conjuntivite. Entretanto, alguns pacientes mais vulneráveis podem desenvolver quadros de insuficiência respiratória aguda com pneumonia associada e quadros tromboembólicos, necessitando em algumas situações de cuidados de terapia intensiva.

A maioria das pessoas (cerca de $80 \%$ ) se recupera da doença sem precisar de tratamento hospitalar. Entretanto, uma em cada seis pessoas infectadas por COVID-19 fica gravemente doente e desenvolve dificuldade de respirar, sendo principalmente essas pessoas portadoras de comorbidades.

Com base nestes grupos de pacientes mais vulneráveis às formas graves da doença, 0 Ministério da Saúde (MS) determinou que alguns grupos de risco devem ser resguardados quanto ao contato com possíveis pacientes sintomáticos. São parte dos grupos de risco os idosos, grávidas, crianças menores de 5 anos, população indígena aldeada, portadores de doenças cardiovasculares, incluindo hipertensão, diabéticos, obesos, portadores de doença pulmonar crônica (incluindo asma e tuberculose), doentes renais crônicos, hepatopatas, portadores de doenças hematológicas, portadores de imunodeficiências ou alterações cromossômicas e portadores de transtornos neurológicos que comprometem a função pulmonar (Ministério da Saúde, 2020).

O fato verdadeiro é que a alta transmissibilidade do coronavírus e a ausência de imunobiológicos disponíveis ensejam ações de monitoramento, avaliação e pesquisa sociodemográfica para se conhecer o espectro da doença e seu mecanismo patogênico numa população, melhorando a eficiência de dados de incidência e prevalência da COVID-19. Neste sentindo, justifica-se numa comunidade acadêmica um diagnóstico preciso quanto à compreensão do impacto das iniquidades em saúde nos grupos vulneráveis, permitindo uma abordagem de 
prevenção que implemente políticas organizacionais e institucionais com vistas ao alcance de medidas eficazes para salvar vidas. (Kang \& Jung, 2020; Wang, et al., 2020)

Outro fato importante é o comportamento letal da COVID-19 especificamente por idades associado a outras causas importantes como doenças crônicas que geralmente apresentam-se em idades mais avançadas (European Commission, 2020). Assim, mapear como essas populações organicamente mais vulneráveis se relacionam com nossas atividades institucionais é imprescindível, seja por uma relação direta do vínculo institucional, seja indireta com alguém da comunidade acadêmica como elo de transmissão.

\section{4 - A COVID-19 no contexto educacional:}

Com o advento da pandemia da COVID-19, uma das principais recomendações em todos os países nos quais a doença atingiu números expressivos foi evitar a permanência em locais públicos com grande concentração de pessoas, como transportes públicos de massa, teatros, cinemas, centros comerciais, escritórios, estabelecimentos de ensino e restaurantes (Rodríguez-Morales et al., 2020). Por serem as escolas ambientes em que há grande circulação de alunos, familiares e funcionários, torna-se um alvo importante da disseminação de viroses. O fechamento das escolas foi, então, tomado como medida emergencial de saúde, sendo estes um dos primeiros locais a fecharem suas portas e decretarem suspensas as atividades de ensino presencial.

A instituição de ensino deve atuar como local estratégico para implementação de medidas de prevenção, tanto no que diz respeito a medidas de controle da transmissão, com restrição de circulação de pessoas em seu ambiente físico, como também atuar em sua missão de educar, através de campanhas de saúde para sua comunidade acadêmica. O professor possui um papel importantíssimo neste momento enquanto disseminador de boas práticas em saúde e formação de um espírito coletivo de ajuda e respeito às regras sanitárias.

Cervantes Holguín (2020) faz uma revisão da literatura sobre este tema, alertando não só sobre o risco de contaminação da comunidade acadêmica em virtude da pandemia, mas principalmente fazendo uma reflexão sobre as implicações na saúde mental dos estudantes e profissionais da educação que permaneceram sem atividades presenciais, assim como sobre a exclusão digital enquanto lacuna de aprendizagem para estudantes que não tiveram acesso ao ensino remoto, aumentando assim a desigualdade educacional em muitos países. Por isso, a educação online pode apoiar a aprendizagem para muitos alunos, mas precisa ser cuidadosamente projetada e individualizada para não aprofundar essas desigualdades e divisões sociais (Kaden, 2020).

Por outro lado, para os países e segmentos educacionais que conseguiram se adaptar a formas alternativas de ensino, observou-se um crescimento exponencial no desenvolvimento de programas e softwares de ajuda a professores e alunos, com estratégias alternativas e metodologias ativas de ensino centradas no aluno enquanto buscador do seu próprio conhecimento. 


\section{METODOLOGIA}

Trata-se de um estudo transversal, observacional e descritivo para avaliação de dados sociodemográficos e de saúde da comunidade acadêmica do IFRN.

É um estudo com características qualitativas (visto que as significações dos fenômenos do processo saúde-doença são essenciais para melhorar a qualidade da relação paciente-famíliainstituição) (Turato, 2005) e quantitativas (por basear-se em pesquisa com questionário e na qual os resultados são tabulados através de gráficos e tabelas, após realização de análise estatística); transversal, pois a pesquisa refere-se à doença ou sintomas que o entrevistado possui no mesmo momento em que a pesquisa é feita; observacional, pois não há intenção de realizar nenhuma intervenção com a amostra, apenas observar as características sócio-demográficas e de saúde desta população de estudo; e descritivo, pois não se almeja comprovar nenhuma hipótese, apenas descrever os fatos e achados (Campana, 2001; Hochman, 2005).

Com o advento da pandemia da COVID19, as atividades laborais presenciais foram suspensas em todos os campi do IFRN, de modo que se viu a necessidade de avaliar as condições de saúde de toda a comunidade acadêmica, pensando em um possível retorno presencial quando este fosse determinado com segurança. Foi então pensado neste instrumento de pesquisa como uma forma de subsidiar tomadas de decisão quando de um retorno à modalidade presencial de trabalho ou manutenção de atividades remotas para grupos específicos. A pesquisa foi direcionada a todos os servidores (docentes e técnico-administrativos), estagiários, alunos e funcionários terceirizados dos 22 campi e reitoria do IFRN.

Foi realizada a coleta de dados a partir de um formulário eletrônico enviado para a toda a comunidade acadêmica do IFRN (servidores, discentes, estagiários e funcionários terceirizados) sob o nome "Análise de Saúde da Comunidade Acadêmica do IFRN" (ASA IFRN), com link disponibilizado nos meios de comunicação institucionais (Instagran, grupos de WhatsApp, e-mail institucional e a partir da página do SUAP -Sistema Unificado de Administração Pública). O SUAP consiste em um sistema desenvolvido pela equipe da Diretoria de Gestão de Tecnologia da Informação do IFRN para a gestão dos processos administrativos internos da instituição, ao qual todos os servidores e alunos ativos têm acesso. Para os funcionários terceirizados que não tinham acesso ao SUAP, foi disponibilizado o questionário semelhante por meio do Google Forms.

Foram incluídos no estudo todos os servidores (docentes e técnico-administrativos), estagiários, alunos e funcionários terceirizados do IFRN que aceitaram voluntariamente participar da pesquisa, respondendo ao questionário.

Atualmente o IFRN possui um universo populacional de 36.193 pessoas, sendo 3381 servidores ativos e estagiários, 32.136 alunos regularmente matriculados e 676 funcionários terceirizados, distribuídos por 22 campi e reitoria. Deste total, 14.484 pessoas responderam ao ASA, perfazendo um percentual de $40 \%$ da população, número considerado satisfatório tendo em vista que nem todos os membros da comunidade acadêmica possuem acesso à internet, além das dificuldades encontradas em razão do afastamento ocasionado pela pandemia. O número de 
respondentes ficou assim distribuído: $60,4 \%$ do total de servidores, $37,1 \%$ do total de alunos e $75,6 \%$ do total de terceirizados.

A coleta de dados ocorreu entre o período de 09 de junho a 21 de julho de 2020. Todos os respondentes concordaram em participar da pesquisa através da anuência do Termo de Consentimento Livre e Esclarecido.

O questionário de pesquisa (ASA IFRN) possuía questões relacionadas a fatores sóciodemográficos, tais como idade, local de moradia, local de trabalho/estudo, cargo, número de cômodos na residência e número de pessoas na residência; e relacionados a saúde, tais como imunização contra influenza, presença de sintomas sugestivos de COVID19, realização de teste diagnóstico para COVID19, existência de comorbidades ou coabitação com pessoas de grupos de risco.

Para fins de definição, foram considerados grupos de risco os respondentes que se enquadravam nos seguintes critérios estabelecidos pelo Ministério da Saúde (2020): idosos, grávidas, crianças menores de 5 anos, população indígena aldeada, portadores de doenças cardiovasculares, incluindo hipertensão, diabéticos, obesos, portadores de doença pulmonar crônica (incluindo asma e tuberculose), doentes renais crônicos, hepatopatas, portadores de doenças hematológicas, portadores de imunodeficiências ou alterações cromossômicas e portadores de transtornos neurológicos que comprometem a função pulmonar. Vale à pena frisar que este dado foi considerado por meio da autodeclaração, não tendo sido exigida a apresentação de nenhum documento médico comprobatório.

Da mesma forma, na pergunta referente à positividade do exame para COVID-19, foram consideradas como positivas as respostas autodeclaradas para qualquer um dos exames confirmatórios (RT-PCR para SARS-COV2 ou Sorologia IgM ou IgG para COVID-19). Os sintomas relacionados à doença COVID-19, de acordo com diretrizes do Ministério da Saúde (2020), foram: dor na garganta, fraqueza, dor no corpo, diarreia, tosse seca, coriza, cansaço, dificuldade respiratória, febre alta, dor de cabeça, alteração no olfato, febre e alteração no paladar.

Os dados coletados foram compilados pelo NAVI (Núcleo Avançado em Inovação Tecnológica, que é um laboratório de tecnologias ligadas à inovação localizado no campus NatalCentral do IFRN) e analisados sob a forma de frequências por meio de gráficos e tabelas. A análise estatística foi feita por meio de análise descritiva das frequências e análise bivariada por meio dos testes de Qui-quadrado (quando amostra com distribuição normal) ou Exato de Fischer (quando distribuição não-normal), em virtude das variáveis serem categóricas e dicotômicas, para uma significância de 5\% (ou seja, o menor nível de significância para se rejeitar a hipótese nula é de 5\%, sendo, neste caso, o $p$ valor significativo se $<0,05$ ).

\section{RESULTADOS E DISCUSSÃO}

Sobre dados gerais de saúde dos respondentes, observou-se que $26,77 \%$ possuem acesso a saúde privada, além do SUS (Sistema Único de Saúde) e que 30,94\% haviam tomado a vacina contra H1N1 no ano de 2020. 
Em relação à presença de pessoas enquadradas como grupos de risco, de acordo com os critérios estabelecidos pelo Ministério da Saúde, observou-se que 9,55\% da população possuía algum fator de risco para complicações de COVID-19. As comorbidades dos respondentes estão representadas na Figura 1, sendo as mais prevalentes a Hipertensão arterial, obesidade e Doença pulmonar crônica. Destaca-se que um mesmo paciente pode eventualmente apresentar mais de uma doença.

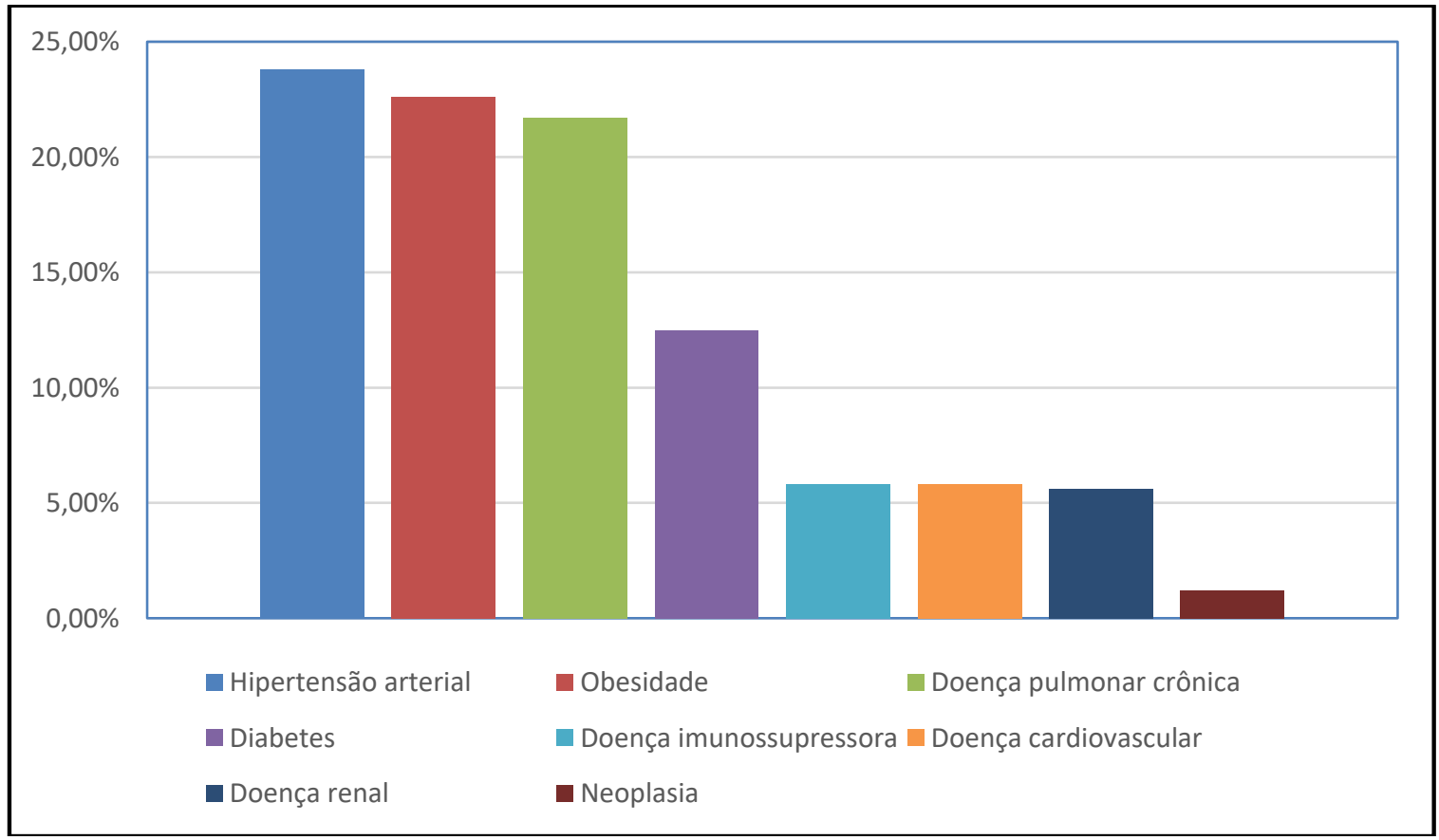

Figura 1: Distribuição das comorbidades na comunidade acadêmica do IFRN. Natal/RN, 2020. Fonte: Dados da pesquisa, 2020.

Levando-se em consideração o mapeamento dos grupos de risco para o retorno presencial às atividades, faz-se necessário identificar estes pontos de fragilidade para que o retorno seja seguro para quem pode retornar e mantenha-se o trabalho/estudo à distância para quem está amparado por lei. Os alunos, em detrimento de terem sido os que mais responderam à pesquisa, são pouco acometidos por situações consideradas de risco para COVID19 (apenas 8,2\% dos alunos possuem comorbidades e $0,01 \%$ possuem 60 anos ou mais).

Quanto aos servidores/estagiários, observa-se também uma população jovem no IFRN, sendo que apenas $1,9 \%$ dos servidores possuem 60 anos ou mais, com uma média de idade em 39,26 anos. Apesar disso, no tocante às comorbidades, observou-se que $22 \%$ dos servidores possuem alguma doença enquadrada nos grupos de risco. Este dado é relevante no que diz respeito a ações de intervenção na saúde do servidor, implementação de medidas de prevenção e qualidade de vida no trabalho e fora dele. Por fim, quando os dados são analisados do ponto de vista dos funcionários terceirizados, identificou-se que $2,4 \%$ destes eram idosos ( $\geq 60$ anos) e $7 \%$ possuíam comorbidades. 
A análise preliminar destes dados nos remete a um cuidado especial com os servidores, visto que estes são a maior parcela dentre os grupos de risco, sugerindo que aproximadamente $1 / 4$ dos servidores mantenham-se em trabalho remoto quando do retorno presencial.

Um total de 1.733 pessoas (11,96\% dos respondentes) afirmaram ter apresentado sintomas de COVID-19, sendo estes exibidos na Figura 2. Nota-se que os sintomas mais frequentemente declarados foram a cefaleia (dor de cabeça), dor de garganta e cansaço, e que cada paciente pode ter sido acometido por mais de um sintoma simultaneamente. Destes sintomáticos, 239 (1,71\%) confirmaram a doença por meio de algum tipo de testagem laboratorial, sendo que a maioria foi de alunos (Figura 3).

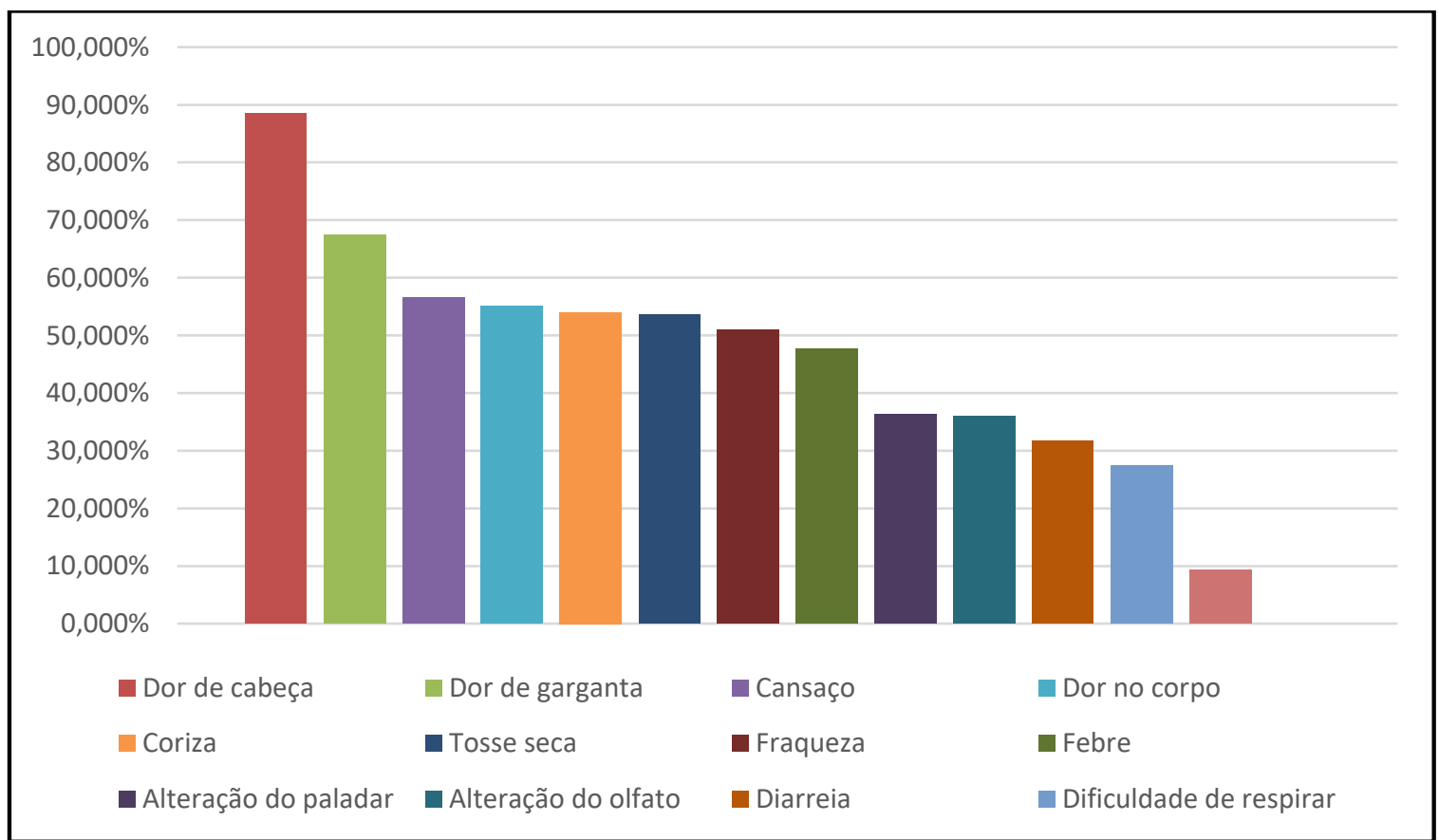

Figura 2: Distribuição dos sintomas de COVID-19 na comunidade acadêmica do IFRN. Natal/RN, 2020. Fonte: Dados da pesquisa, 2020. 


\section{Casos confirmados de COVID-19 distribuidos por vínculo}

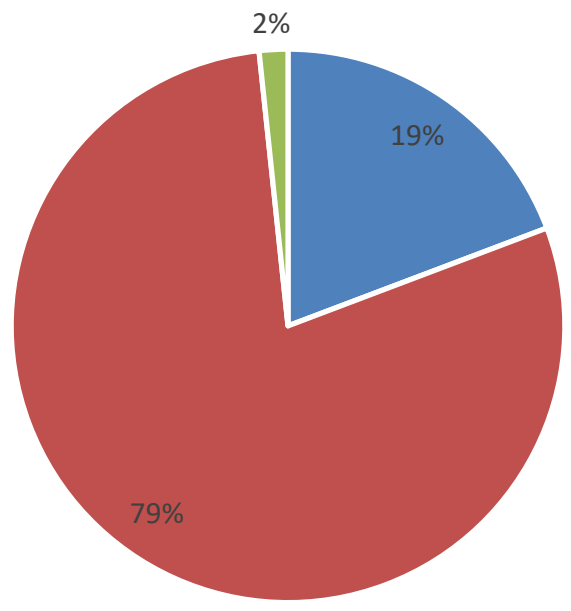

- Servidores Alunos - Terceirizados

Figura 3: Distribuição dos casos confirmados laboratorialmente de COVID-19 na comunidade acadêmica do IFRN. Natal/RN, 2020.

Fonte: Dados da pesquisa, 2020.

Referente a dados sociais, importantes na análise de potencial de transmissibilidade da doença, a pesquisa identificou que $61,25 \%$ dos respondentes residem com pessoas de grupos de risco, sendo a maioria alunos (Figura 4); a maioria mora em residências com mais 3 a 4 pessoas, sendo que a maioria das residências possuem 4 ou mais cômodos. Estes dados são importantes visto que, mesmo que o aluno não apresente risco potencial de letalidade ou complicações da doença para si, pode levar este risco para seus familiares/coabitantes, sendo estes grandes vetores justamente pelo fato dos jovens em geral desenvolverem quadros leves da doença ou mesmo serem assintomáticos, e com isso gerarem risco para seus familiares (Zou, et al., 2020). Na tomada de decisão por um isolamento social mais rígido, deverão ser prioridades os que coabitam com indivíduos de grupo de risco e com número de cômodos no domicílio reduzido. 


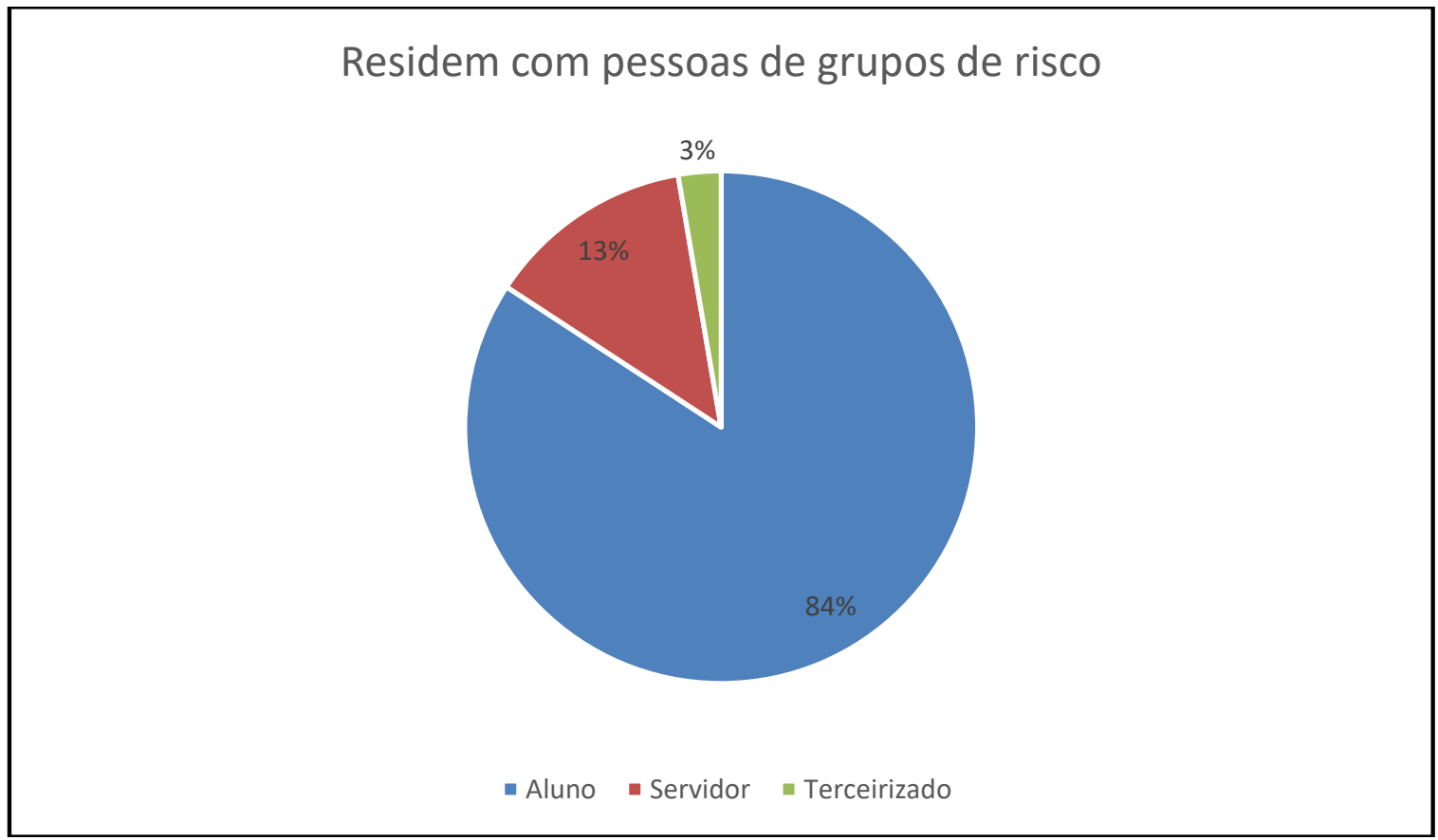

Figura 4: Distribuição dos respondentes quanto a coabitação com pessoas de grupos de risco, divididos por vínculo. Natal/RN, 2020.

Fonte: Dados da pesquisa, 2020.

Sobre a localidade de trabalho ou estudo, $49,84 \%$ da comunidade acadêmica transitam entre municípios, sendo a maioria de alunos (moram em um município e estudam ou trabalham em outro), sendo que $86,42 \%$ fazem esse deslocamento diariamente. Este dado reflete a possibilidade do fluxo de pessoas intermunicípios gerar influências na taxa de transmissibilidade de cada município, visto que mesmo que o indivíduo seja originário de um município com baixa taxa de transmissibilidade, no momento em que o mesmo transita por locais com altos índices de infecção, este estará mais exposto ao risco e, pior, poderá levar a doença para sua localidade de origem (Bruns, Kragulijac, \& Bruns, 2020).

Quando avaliados os pacientes de grupos de risco que apresentaram sintomas de COVID-19, observamos associações importantes identificadas nas Tabelas 1 e 2. A obesidade foi identificada como a comorbidade com mais sintomas associados (tosse, cansaço, coriza, dificuldade respiratória, dor de garganta, dor no corpo, cefaleia, diarreia e fraqueza) e o cansaço foi o único sintoma que esteve associado com todas as comorbidades avaliadas (hipertensão arterial, diabetes, obesidade, doença pulmonar crônica, doença imunossupressora, doença cardiovascular, doença renal crônica e neoplasia).

Tabela 1: Associações entre os sintomas de COVID-19 e hipertensão arterial, diabetes, obesidade e doença pulmonar crônica. Natal/RN, 2020.

\begin{tabular}{l|l|l|l|l}
\hline & $\begin{array}{l}\text { Hipertensão } \\
\text { arterial }\end{array}$ & Diabetes & Obesidade & $\begin{array}{l}\text { Doença pulmonar } \\
\text { crônica }\end{array}$ \\
\hline Febre & $p=0,087$ & $p=0,148$ & $p=0,120$ & $p=0,828$ \\
\hline
\end{tabular}




\begin{tabular}{|c|c|c|c|c|}
\hline Tosse & $\begin{array}{l}p=0,041 \\
\text { OR }=1,435 \\
I C=1,013-2,032\end{array}$ & $\begin{array}{l}p=0,039 \\
\text { OR }=1,604 \\
I C=1,019-2,523\end{array}$ & $\begin{array}{l}p<0,001 \\
\text { OR }=2,117 \\
I C=1,555-2,882\end{array}$ & $\begin{array}{l}p<0,001 \\
O R=1,957 \\
I C=1,415-2,704\end{array}$ \\
\hline Cansaço & $\begin{array}{l}p<0,001 \\
\text { OR }=1,765 \\
I C=1,287-2,420\end{array}$ & $\begin{array}{l}p=0,001 \\
\text { OR }=1,948 \\
I C=1,289-2,944\end{array}$ & $\begin{array}{l}p<0,001 \\
\text { OR }=2,065 \\
I C=1,521-2,802\end{array}$ & $\begin{array}{l}p<0,001 \\
O R=2,611 \\
I C=1,957-3,484\end{array}$ \\
\hline Coriza & $p=0,315$ & $p=0,518$ & $\begin{array}{l}p<0,001 \\
\text { OR }=1,905 \\
I C=1,384-2,624\end{array}$ & $\begin{array}{l}p=0,009 \\
O R=1,589 \\
I C=1,120-2,252\end{array}$ \\
\hline $\begin{array}{l}\text { Dificuldade } \\
\text { respiratória }\end{array}$ & $\begin{array}{l}p=0,002 \\
\text { OR }=1,900 \\
I C=1,245-2,901\end{array}$ & $p=0,120$ & $\begin{array}{l}p=0,016 \\
\text { OR }=1,728 \\
I C=1,102-2,708\end{array}$ & $\begin{array}{l}p<0,001 \\
O R=3,813 \\
I C=2,710-5,364\end{array}$ \\
\hline $\begin{array}{l}\text { Dor de } \\
\text { garganta }\end{array}$ & $\begin{array}{l}p=0,030 \\
\text { OR }=1,419 \\
I C=1,033-1,949\end{array}$ & $p=0,073$ & $\begin{array}{l}p<0,001 \\
\text { OR }=2,039 \\
I C=1,532-2,714\end{array}$ & $\begin{array}{l}p=0,005 \\
O R=1,579 \\
I C=1,147-2,172\end{array}$ \\
\hline $\begin{array}{l}\text { Alteração } \\
\text { paladar }\end{array}$ & $p=0,058$ & $\begin{array}{l}p=0,019 \\
O R=1,832 \\
I C=1,095-3,065\end{array}$ & $p=0,907$ & $p=0,462$ \\
\hline $\begin{array}{l}\text { Alteração } \\
\text { olfato }\end{array}$ & $p=0,349$ & $p=0,314$ & $p=0,687$ & $p=0,853$ \\
\hline $\begin{array}{ll}\text { Dor } & \text { no } \\
\text { corpo } & \end{array}$ & $\begin{array}{l}p=0,004 \\
\text { OR }=1,623 \\
I C=1,168-2,254\end{array}$ & $p=0,051$ & $\begin{array}{l}p=0,002 \\
\text { OR }=1,667 \\
I C=1,195-2,325\end{array}$ & $p=0,101$ \\
\hline Cefaleia & $p=0,116$ & $p=0,083$ & $\begin{array}{l}p<0,001 \\
O R=1,785 \\
I C=1,365-2,334\end{array}$ & $\begin{array}{l}p=0,014 \\
O R=1,443 \\
I C=1,076-1,935\end{array}$ \\
\hline Diarreia & $\begin{array}{l}p=0,006 \\
\text { OR }=1,751 \\
I C=1,166-2,629\end{array}$ & $p=0,169$ & $\begin{array}{l}p<0,001 \\
O R=2,423 \\
I C=1,679-3,497\end{array}$ & $p=0,061$ \\
\hline Fraqueza & $p=0,532$ & $p=0,268$ & $\begin{array}{l}p<0,001 \\
\text { OR }=1,788 \\
I C=1,281-2,495\end{array}$ & $p=0,357$ \\
\hline
\end{tabular}

OR: odds ratio. IC: Intervalo de confiança. $p$ : $p$-value.

Tabela 2: Associações entre os sintomas de COVID-19 e Doença imunossupressora, Doença cardiovascular, Doença renal crônica e Neoplasia. Natal/RN, 2020.

\begin{tabular}{l|l|l|l|l}
\hline & $\begin{array}{l}\text { Doença } \\
\text { imunossupressora }\end{array}$ & $\begin{array}{l}\text { Doença } \\
\text { cardiovascular }\end{array}$ & $\begin{array}{l}\text { Doença renal } \\
\text { crônica }\end{array}$ & Neoplasia \\
\hline Febre & $p=0,148$ & $\begin{array}{l}p=0,005 \\
\mathrm{OR}=2,296 \\
\mathrm{IC}=1,256-4,199\end{array}$ & $\begin{array}{l}p=0,042 \\
\mathrm{OR}=1,947 \\
\mathrm{IC}=1,012-3,749\end{array}$ & $p=0,850$ \\
\hline Tosse & $p=0,059$ & $\begin{array}{l}p=0,006 \\
\mathrm{OR}=2,214 \\
\mathrm{IC}=1,236-3,964\end{array}$ & $p=0,426$ & $p=0,145$ \\
& & $p=0,003$ & $p=0,007$ & \\
\hline Cansaço & $p=0,011$ & $\mathrm{OR}=2,299$ & $\mathrm{OR}=2,200$ & $\begin{array}{l}\text { OR }=3,200 \\
\mathrm{IC}=1,308-4,043\end{array}$ \\
& $\mathrm{OR}=2,090$ & $p=1,277-3,945$ & $\mathrm{IC}=1,091-9,382$ \\
\hline Coriza & $\mathrm{IC}=1,168-3,740$ & $\mathrm{IC}=0,022$ & $p=0,133$ & $p=0,147$ \\
& $\mathrm{OR}=1,995$ & & & \\
& $\mathrm{IC}=1,092-3,645$ & & & \\
\hline
\end{tabular}




\begin{tabular}{|c|c|c|c|c|}
\hline $\begin{array}{l}\text { Dificuldade } \\
\text { respiratória }\end{array}$ & $\begin{array}{l}p=0,035 \\
O R=2,244 \\
I C=1,038-4,852\end{array}$ & $\begin{array}{l}p=0,033 \\
O R=2,265 \\
I C=1,047-4,899\end{array}$ & $p=0,096$ & $p=0,707$ \\
\hline $\begin{array}{l}\text { Dor de } \\
\text { garganta }\end{array}$ & $\begin{array}{l}p=0,009 \\
O R=2,047 \\
I C=1,185-3,539\end{array}$ & $p=0,123$ & $p=0,357$ & $p=0,291$ \\
\hline $\begin{array}{l}\text { Alteração } \\
\text { paladar }\end{array}$ & $p=0,196$ & $p=0,166$ & $\begin{array}{l}p=0,003 \\
O R=2,636 \\
I C=1,367-5,081\end{array}$ & $p=0,240$ \\
\hline $\begin{array}{l}\text { Alteração } \\
\text { olfato }\end{array}$ & $p=0,072$ & $p=0,160$ & $\begin{array}{l}p=0,044 \\
O R=2,074 \\
I C=1,004-4,285\end{array}$ & $p=0,600$ \\
\hline $\begin{array}{ll}\text { Dor } & \text { no } \\
\text { corpo } & \end{array}$ & $\begin{array}{l}p=0,001 \\
\mathrm{OR}=2,524 \\
\mathrm{IC}=1,459-4,365\end{array}$ & $\begin{array}{l}p=0,002 \\
O R=2,353 \\
I C=1,338-4,137\end{array}$ & $\begin{array}{l}p=0,017 \\
O R=2,054 \\
I C=1,122-3,758\end{array}$ & $\begin{array}{l}p=0,047 \\
\text { OR }=3,273 \\
I C=1,116-9,597\end{array}$ \\
\hline Cefaleia & $p=0,132$ & $\begin{array}{l}p=0,012 \\
O R=1,902 \\
I C=1,145-3,159\end{array}$ & $p=0,091$ & $p=0,576$ \\
\hline Diarreia & $p=0,535$ & $\begin{array}{l}p=0,006 \\
O R=2,530 \\
I C=1,272-5,030\end{array}$ & $p=0,073$ & $p=0,194$ \\
\hline Fraqueza & $p=0,231$ & $p=0,100$ & $\begin{array}{l}p=0,001 \\
O R=2,625 \\
I C=1,491-4,625\end{array}$ & $p=0,139$ \\
\hline
\end{tabular}

OR: Odds ratio. IC: Intervalo de confiança. $p$ : $p$-value.

A obesidade já foi bem associada com formas graves e complicadas de COVID-19, e definitivamente este grupo merece toda atenção desde as medidas preventivas como isolamento mais rígido e atividades remotas. O fato de estar associada a vários sintomas remete à implicação multissistêmica que o vírus desenvolve em pacientes obesos, provavelmente devido à síndrome metabólica associada e ao estado hiperinflamado que estes pacientes possuem. Também se verifica a maior necessidade de suporte avançado respiratório entre os pacientes obesos graças ao efeito negativo da obesidade no volume, função e expansão pulmonar, além da magnitude de inflamação pulmonar nestes pacientes. (Huang, et al., 2020)

$O$ retorno às atividades presenciais com uma pandemia em curso foi realidade em muitos países, sendo a permissão para estudantes e funcionários em risco permanecerem em casa, uma condição fundamental para a segurança destes, bem como garantir que todos os casos suspeitos ou confirmados de COVID-19 sejam imediatamente colocados em quarentena. Além disso, ensino à distância e planos de continuidade serão primordiais em situações nas quais as escolas precisam fechar temporariamente para impedir a propagação do vírus. (Melnick \& Darling-Hammond, 2020).

A pesquisa apresentou algumas limitações, tais como o número de respondentes ter sido desigual entre as categorias (servidores, estagiários, terceirizados e alunos) e entre os campi (alguns campi tiveram mais adesão que outros); o fato de que os sintomas e diagnósticos refletiram apenas aquele momento específico da pesquisa, de modo que dias ou meses depois estes números de infectados possivelmente já serão maiores; o fato de muitos respondentes não terem realizado 
exames confirmatórios da COVID19, mas mesmo assim os sintomas de síndrome gripal terem sido enquadrados na pesquisa com base epidemiológica; e por fim, o fato de que o diagnóstico confirmado por exame foi aceito apenas por auto-declaração, os pesquisadores não tiveram acesso aos exames laboratoriais dos pacientes.

\section{CONCLUSÃO}

Num cenário de extrema incerteza como o que tem sido vivenciado pelas instituições a partir do estabelecimento da pandemia do novo coronavírus, ter informações sobre o perfil epidemiológico da comunidade que compõe uma instituição extremamente capilar como é o caso do IFRN, pode se configurar como um diferencial para obtenção de sucesso nas estratégias para manutenção do funcionamento da instituição e para o planejamento das fases de retomada das atividades presenciais, incluindo neste estudo modelos de cenários, população que será contemplada com as formas em que as atividades de trabalho possam vir a ser desenvolvidas (trabalho remoto, turnos alternados de revezamento ou flexibilização do horário de início e término da jornada de trabalho) e também as características de saúde e epidemiológicas ( tais como: índices de ocupação dos leitos de UTIs e a taxa de transmissibilidade) que venham a balizar as fases de retomada.

Desta forma, a realização desta pesquisa entrega subsídios que podem auxiliar os gestores da instituição na tomada de decisões acerca dos rumos do IFRN face ao desenvolvimento da pandemia, uma vez que conhecer a realidade do ponto de vista social e as condições de saúde dos públicos que compõe o IFRN, considerando-se as comorbidades associadas ao risco de agravamento na evolução do curso da COVID-19, assim como o percentual de indivíduos já acometidos pela doença, por exemplo, permite desenvolver estratégias mais efetivas de proteção e mitigação dos riscos aos quais os indivíduos que formam a organização estão expostos.

Entre os achados da pesquisa, os servidores/estagiários do IFRN formam o grupo identificado como os que mais apresentam fatores de risco para as formas graves de COVID-19, devendo estes receber um olhar especial quando do retorno às atividades presenciais. Hipertensão e obesidade foram as comorbidades mais prevalentes. Sabendo-se serem essas importantes entidades clínicas relacionadas a casos graves de COVID-19, faz-se necessário um olhar diferenciado para estes servidores, com permanência de trabalho remoto se possível, assim como instituição de atividades de qualidade de vida com vistas à educação em saúde e prevenção destes agravos nos servidores saudáveis.

A maioria dos indivíduos positivados para COVID-19 foi de alunos, sendo os sintomas mais frequentes a cefaleia, dor de garganta e cansaço. Apesar de representarem baixo risco de desenvolver formas graves da doença por serem jovens, os alunos também devem ser contemplados com uma avaliação específica quando houver o retorno presencial às aulas pois grande parte deles coabitam com pessoas idosas ou de grupos de risco, portanto, podem ser vetores para estas pessoas.

Por fim, a obesidade foi identificada como a comorbidade com mais sintomas associados e o cansaço foi o sintoma que esteve associado com todas as comorbidades. Logo, ações de educação 
em saúde e prevenção com pessoas obesas da nossa comunidade acadêmica devem ser instituídas para evitar complicações em caso de adquirir COVID-19.

O conhecimento acerca de variáveis como as mencionadas anteriormente, representa um achado fundamental para que seja possível planejar aspectos como as fases da retomada das atividades presenciais na Instituição com maior precisão. Reconhecer as comorbidades mais comuns entre os servidores, por exemplo, pode ofertar subsídios que norteiem a formulação de políticas de atenção à saúde mais efetivas. Além disso, a mitigação dos riscos aos quais a comunidade do IFRN que compõe o grupo que necessita de maiores cuidados quanto à contaminação com a COVID- 19 exige diagnóstico para subsidiar o planejamento. No IFRN, entendese que é necessário definir grupos elegíveis para as etapas iniciais de retomada presencial no ensino, atividades técnicas e administrativas.

Por fim, ressaltamos que por se tratar de doença em pleno curso de pandemia, os quadros podem se alterar muito rapidamente e assim, sugere-se que uma nova etapa deste mesmo estudo seja realizada num momento mais próximo ao retorno às atividades presenciais, para que se possa obter um panorama atualizado do quantitativo de pessoas que já foram infectadas e quais medidas de restrição necessitam ser tomadas com pacientes dos grupos de risco. Espera-se, ainda, que com o seguimento da pesquisa, mais pessoas possam participar e responder ao questionário.

\section{REFERÊNCIAS}

Arruda, E. P. (15 de Maio de 2020). EDUCAÇÃO REMOTA EMERGENCIAL: elementos para políticas públicas na educação brasileira em tempos de Covid-19. Em Rede: Revista de Educação à distância, 7(1), pp. 257-275. Fonte: https://www.aunirede.org.br/revista/index.php/emrede/article/view/621

Barata, R. d. (jan/fev de 1987). Epidemias. Cadernos de Saúde Pública, pp. 9-15.

Brasil . (6 de fevereiro de 2020). Lei 13979, de 6 de fevereiro de 2020. Fonte: Diário Oficial da União: https://www.in.gov.br/en/web/dou/-/lei-n-13.979-de-6-de-fevereiro-de-2020-242078735

Bruns, D. P., Kragulijac, N. V., \& Bruns, T. R. (21 de Abril de 2020). COVID-19: Facts, Cultural Considerations, and risk of stigmatization. Journal of Transcultural Nursing. doi:https://doi.org/10.1177/1043659620917724

Campana, A. O., Padovani, C. R., laria, C. T., Freitas, C. B. D., De Paiva, S. A. R. \& Hossne, W. S. (2001). Investigação científica na área médica. 1st ed. Sao Paulo: Manole.

Cervantes Holguín, E., \& Gutiérrez Sandoval, P. R. (2020). Resistir la Covid-19. Intersecciones en la Educación de Ciudad Juárez, México. Revista Internacional De Educación Para La Justicia Social, 9(3), 7-23. https://doi.org/10.15366/riejs2020.9.3.001

European Commission. (4 de Maio de 2020). COVID-19 Cases and case fatality rate by age. Fonte: European Commission: https://ec.europa.eu/knowledge4policy/publication/covid-19-casescase-fatality-rate-age_en 
Governo do Estado do Rio Grande do Norte. (01 de abril de 2020). Decreto 29583, de 1o de abril de 2020. Fonte: Diário Oficial do Estado do RN: http://diariooficial.rn.gov.br/dei/dorn3/docview.aspx?id_jor=00000001\&data=20200402\&id_ doc $=678994$

Harvey, D., Zizek, S., Baidou , A., Davis, M., Bihr, A., \& Zibechi, R. (2020). Coronavírus e a luta de classes. Brasil: Terra sem Amos.

Hochman, B., Nahas, F. X., Oliveira Filho, R. S., \& Ferreira, L. M. (2005) Desenhos de pesquisa. Acta Cir. Bras. [online]. vol.20, suppl.2 [cited 2020-10-27], pp.2-9. Available from: $<$ http://www.scielo.br/scielo.php?script=sci_arttext\&pid=S0102-

$86502005000800002 \&$ Ing=en\&nrm=iso>. ISSN 1678-2674. https://doi.org/10.1590/S010286502005000800002 .

Huang, J.-F., Wang, X.-B., Zeng, K. I., Liu, W.-Y., cHEN, J.-J., Gerge, J., \& Zheng, M.-H. (22 de Abril de 2020). Letter to the Editor: Obesity hypoventilation syndrome and severe COVID-19. Metabolism, 108, pp. 1-3. https://linkinghub.elsevier.com/retrieve/pii/S002604952030113X

InfoEscola. (25 de setembro de 2020). Endemia, epidemia e pandemia. Fonte: InfoEscola: https://www.infoescola.com/doencas/endemia-epidemia-e-pandemia/

Jornal da USP. (16 de Abril de 2020). A origem do Sars-CoV-2. Fonte: PFARMA: https://pfarma.com.br/coronavirus/5439-origem-covid19.html

Kaden, U. COVID-19 School Closure-Related Changes to the Professional Life of a K-12 Teacher. Educ. Sci. 2020, 10, 165.

Kang, S.-J., \& Jung, S. (25 de Junho de 2020). Age-Related Morbidity and Mortality among Patients with COVID-19 . Infection \& Chemoterapy, pp. 154-164.

Melnick , H., \& Darling-Hammond, L. (Maio de 2020). Reabrindo Escolas no Contexto da covid-19: diretrizes de saúde e segurança de outros países. LEARNING POLICY INSTITUTE, pp. 1-13. Fonte: https://drive.google.com/file/d/1kxWw0QpRIDYGgTyRvSOZKYOUYq9xeE8H/view

Merchán-Hamann, E. (setembro de 1997). Diagnóstico macrorregional da situação das endemias das Regiões Norte e Nordeste. Informe Epidemiológico do SUS, pp. 43-114.

Ministério da Saúde. (20 de Setembro de 2020a). Linha do Tempo Coronavírus . Fonte: Ministério da Saúde : https://coronavirus.saude.gov.br/linha-do-tempo/

Ministério da Saúde. (2020b). Protocolo de manejo clínico da Covid-19 na atenção especializada. Fonte: Ministério da Saúde https://bvsms.saude.gov.br/bvs/publicacoes/manejo_clinico_covid19_atencao_especializada.pdf

Ministério da Saúde. (01 de setembro de 2020c). Sobre a doença. Fonte: Ministério da Saúde: https://coronavirus.saude.gov.br/sobre-a-doenca\#o-que-e-covid 
Organização Panamericana de Saúde. (11 de março de 2020). OMS afirma que covid agora é caracterizada como pandemia. Fonte: OPAS Brasil: https://www.paho.org/bra/index.php?option=com_content\&view=article\&id=6120:omsafirma-que-covid-19-e-agora-caracterizada-como-pandemia\&Itemid=812

Rodrigues, L. (29 de março de 2020). Conheça as 5 maiores pandemias da história. Fonte: Revista Galileu: https://revistagalileu.globo.com/Ciencia/Saude/noticia/2020/03/conheca-5-maiorespandemias-da-historia.html

Rodríguez-Morales, A. J., Sánchez-Duque, J. A., Hernández, S., Pérez-Díaz, C. E., Villamil-Gómez, W. E., Méndez, C. A., . . . Escalera-Antezana, J. P. (2020). Preparación y control de la enfermedad por coronavirus 2019 (Covid-19) en América Latina. Acta Médica Peruana,37(1), 37. https://doi.org/10.35663/amp.2020.371.909

Silva, L. J. (jan/fev de 2003). O controle das endemias no Brasil e sua história . Ciência e Cultura, pp. 44-47.

Turato, E. R. (2005). Métodos qualitativos e quantitativos na área da saúde. Revista de Saúde Pública, 39 (3), pp. 507-514.

Wang, M. L., Berhman, P., Dulin, A., Baskin, M. L., Buscemi, J., Alcaraz, K. I., . . F Fitzgibbon, M. (16 de Junho de 2020). Addressing inequities in COVID-19 morbidity and mortality: research and policy recommendations. Translational Behavioral Medicine, pp. 516-519.

World Health Organization. (24 de fevereiro de 2010). Emergencies preparedness, response. Fonte: WHO: https://www.who.int/csr/disease/swineflu/frequently_asked_questions/pandemic/en/

Zou, L., Ruan, F., Huang, M., Liang, L., Huang, H., Hong, Z., ... Wu, J. (19 de Fevereiro de 2020). SARSCoV-2 Viral Load in Upper Respiratory Specimens of Infected Patients. The New England Journal of Medicine.

\section{COMO CITAR ESTE ARTIGO:}

Ferreira, L. M. B. M., Morais, D. S. V. D., Oliveira, V. R. C., Nascimento, T. A. R., Silva, P. K. M. (2020). Perfil epidemiológico da comunidade acadêmica do IFRN em relação à covid-19 e análise dos fatores de risco para o retorno presencial às aulas. Holos. 36(5), 1-19.

\section{SOBRE OS AUTORES}

\section{M. B. M. FERREIRA}

Graduação em Medicina pela UFRN (2003); Residência em Otorrinolaringologia pela ESP-CE (2007); Especialista em Geriatria pela ESP-CE (2006); Especialista em Medicina do Trabalho pela Faculdade Estácio de Sá (2010); Mestre em Saúde Coletiva pela UFRN (2013); Doutora em Saúde Coletiva pela UFRN (2016); Médica do IFRN (desde 2008); Professora Adjunta do Departamento de Cirurgia da UFRN - área otorrinolaringologia (desde 2017). E-mail: lidiane.ferreira@ifrn.edu.br ORCID ID: https://orcid.org/0000-0002-2191-8641

\section{S. V. D. MORAIS}

Graduação em Enfermagem pela Universidade Potiguar (2009), pós graduação em Enfermagem do Trabalho 
(2010). Servidora pública federal do Instituto Federal de Roraima (IFRR), ocupante do cargo Técnico Administrativo em Educação-Enfermeira. E-mail: dalyanne.morais@ifrn.edu.br

ORCID ID: https://orcid.org/0000-0002-8457-4196

\section{R. C. OLIVEIRA}

Mestre em Serviço Social pela Universidade Federal do Rio Grande do Norte (2009). Graduada em Serviço Social (2001) e em Pedagogia (2016), ambas as formações pela UFRN. Atualmente é Assistente Social do IFRN, desenvolveu suas atividades na Coordenação de Atenção à Saúde do Servidor (Coass), da Diretoria de Gestão de Pessoas (DIGPE), exercendo as suas atividades atualmente na Diretoria de Gestão de Atividades Estudantis (DIGAE). Atuou como professora da Prefeitura Municipal de Parnamirim, da FAL, da Estácio e UNIFACEX. Tem experiência em pesquisas na área de Serviço Social, com ênfase em Serviço Social, atuando principalmente nos seguintes temas: gênero, tecnologia, segurança, Qualidade de Vida, saúde, mulheres na ciência e educação. E-mail: valeria.carvalho@ifrn.edu.br

ORCID ID: https://orcid.org/0000-0002-8169-4176

\section{T. A. R. NASCIMENTO}

Possui graduação em Odontologia pela Universidade Federal do Rio Grande do Norte (2006), especialização em Vigilância Sanitária pela Faculdade Ateneu- Fortaleza (2010) e mestrado em Demografia pela Univesridade Federal do Rio Grande do Norte (2015). Atualmente doutorando do Programa de Pós Graduação em Ciências da Saúde da Universidade Federal do Rio Grande do Norte. Tem experiência na área de Odontologia e Vigilância Sanitária, atuando principalmente nos seguintes temas: hábitos relacionados à saúde bucal, envelhecimento populacional e Vigilância Sanitária E-mail: thiago.nascimento@ifrn.edu.br ORCID ID: https://orcid.org/0000-0001-8199-9183

\section{P. K. M. SILVA}

Possui graduação em Comunicação Social - Jornalismo pela Universidade Federal do Rio Grande do Norte (2007) e em Marketing pela Estácio (2015). Mestrado em Administração pela Universidade Federal do Rio Grande do Norte (2010) e doutorado em Administração pela Universidade Federal da Paraíba (2018). Atualmente ocupa o cargo de Redator no Instituto Federal de Educação, Ciência e Tecnologia do Rio Grande do Norte. Possui experiência nas áreas de comunicação corporativa, desenvolvendo atividades de assessoria de empresas e instituições, bem como de realização de eventos, e ainda, audiovisual, tendo atuado como repórter de rádio e TV e ainda com a execução de projetos de produção de conteúdos institucionais. Como docente atua principalmente nos seguintes temas: Marketing e Comunicação. E-mail: patricia.mesquita@ifrn.edu.br ORCID ID: https://orcid.org/0000-0002-8169-4176

Editor(a) Responsável: Leandro Costa

Pareceristas Ad Hoc: LENINA SILVA E AHIRAM CASTRO

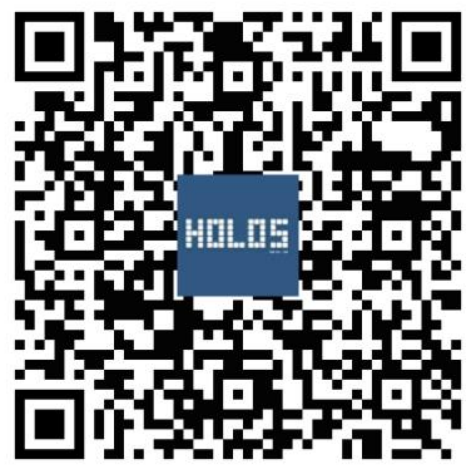

